\title{
Characterization of Treated Multi-Walled Carbon Nanotubes and Antibacterial Properties
}

\author{
Noor. Q. A. Hussan*, Ali A. Taha, Duha S. Ahmed \\ Department of Applied Sciences, University of Technology, Baghdad-Iraq
}

\section{Article information}

\section{Article history:}

Received: April, 15, 2021

Accepted: April, 29, 2021

Available online: June, 25, 2021

Keywords:

R-MWCNTs,

F-MWCNTs,

Functionalized method,

Growth inhibition zone

*Corresponding Author:

Noor. Q. A. Hussan

as.18.70@grad.uotechnology.edu.iq

\begin{abstract}
In the present study, the synthesized Multi-Walled Carbon Nanotubes (MWCNTs) were chemically treated with a mixture of acids to produce functionalized MWNTs. The functionalized MWCNTs were characterized by X-Ray Diffraction Analysis (XRD), Zeta potential and Field-Emission Scanning Electron Microscopy (FE-SEM). The X-ray diffraction reveals the MWCNTs average crystal size of the RMWCNTs and F-MWCNTs were about (3.27 and 3.19) nm, respectively. FESEM images show the formation of R-MWCNTs that appears as cylindrical tubes and aggregated tubes with each other, while the F-MWCNTs appear as less aggregated and tangled clusters than RMWCNTs. Zeta potential measurements showed that the measurement of the R-MWCNT shows a low negative value $-9 \mathrm{mV}$ and F-MWCNT, it was found that the zeta potential value is up to $-29 \mathrm{mV}$. The antibacterial activity was studied against $E$. coli and $P$. aeruginosa bacteria, and indicated the highest growth inhibition zones (IZ) of FMWCNTs as compared with R- MWCNTs against E. coli and Pseudomonas aeruginosa, respectively.
\end{abstract}

\section{Introduction}

Nanotechnology is the combination of science, engineering, and technology that leads to the nanoscale, which is approximately (1 to $100 \mathrm{~nm}$ ). Recently, nanotechnology has become part of many areas of life, such as biomedicine, food, energy, electronics, environment, solar cells, and hydrogen fuel cells. Many researchers mentioned that nanotechnology is the next processing wealth as it shows a good effect on the economy and life in the future [1]. For decades, researchers have worked to improve the performance of metal oxides. Hence, it has become widely used due to good chemical and physical properties such as thermal stability, large surface-to-volume ratio, high mechanical strength and high radiation hardness [2]. One of the carbon allotropes produced from graphite appears as cylindrical tubes with a diameter of up to nanometers and a length of up to millimeter. It is well known that $\mathrm{sp}^{2}$ hybridized carbon forms a planar structure with a complex interlocking that depends on the hexagonal ring arrangement $[3,4]$. Carbon nanotubes (CNTs) possess amazing properties such as chemical stability, mechanical strength, small size, high surface area, high electrical and thermal conductivity [5, 6]. Therefore, attracted researchers' interest for its effective role in many fields such as storage energy, water treatment, thermal conduction, 
catalysts, biomedicine, increasing the strength of polymers and others [7, 8]. Multi-walled carbon nanotubes (MWCNTs) consist of several layers of rolled graphene around each other with a cylindrical shape (concentric tubes). Where the inner diameter of the nanotubes in range (1-3) $\mathrm{nm}$ and the outer diameter in range (2-100) $\mathrm{nm}$ and its length reaches several micrometers $[9,10]$. The structure of multi-walled carbon nanotubes (MWCNTs) is described in two models: The first is called the Russian doll model, which is the wrapping of graphene sheets within a concentric structure. The second model called parchment, which is wrapping a graphene sheet around itself many times like a rolled newspaper [11]. Because they have the largest number of layers, carbon nanotubes can protect the inner tubes from chemical reactions with external materials with high tensile strength, which distinguishes them from Single walled carbon nanotubes (SWCNTs). The interlayer distance between the carbon nanotube layers up to $0.34 \mathrm{~nm}$ is close to the distance between the graphene layers in polyromantic solids. The high ability to decorate with nanoparticles (NPs) and to maintain structure made them attractive to researchers in many fields such as electronic devices, catalysis, biomedicine, biosensors, and electrolysis [12]. Carbon nanotubes (CNTs) have antibacterial properties due to which they inhibit the growth of bacteria. When the bacteria come into physical contact with CNTs, they permeate through the cell membrane, disturb its activity, and demolish the cell viability of bacteria $[6,13]$. To improve the adhesion and bioactive property of functionalized multi walled carbon nanotubes have been chosen because functionalized multiwall carbon nanotubes (F-MWCNTs) exhibit outstanding mechanical, structural, thermal and chemical properties, which act as reinforcement material for high strength complex $[14,15]$. This work involves the treatment and modifying of the R-MWCNTs using the acid treatment. The structural, optical and morphological properties have been identified by a characterization process represented by XRD, FE-SEM and ZETA. On the other hand, the biological activity of the R-MWCNTs and F-MWCNTs was studied by studying the antibacterial activity using diffusion well method against $E$. coli and $P$. aeruginosa bacteria by using different concentrations.

\section{Experimental part}

\subsection{Acid Treatment of $R-M W C N T s$}

In this section, 2 grams of raw-MWCNTs was treated with $200 \mathrm{~mL}$ of an acid mixture of sulfuric acid and nitric acid $(3: 1 \mathrm{v} / \mathrm{v})$ to produce functional groups in treated MWCNTs like $-\mathrm{OH}$ and/ $-\mathrm{COOH}$. The mixture was placed in an ultrasonic bath at temperature $37^{\circ} \mathrm{C}$ for $40 \mathrm{~min}$, then washed with distilled water by using vacuum-filtered through a $\left(0.22 \mu \mathrm{m}\right.$ cellulose nitrate membrane), and dried at $100^{\circ} \mathrm{C}$ for $24 \mathrm{~h}$. afterwards, the resulting F-MWCNTs were treated with hydrogen peroxide $\mathrm{H}_{2} \mathrm{O}_{2}(30 \%)$ by the same procedure in order to complete the oxidative process started by $\mathrm{H}_{2} \mathrm{SO}_{4}$ and $\mathrm{HNO}_{3}$ )[16]. Figure 1 demonstrates schematic diagram of functionalized F-MWCNTs by using an acid treatment in two steps with acid solutions. 


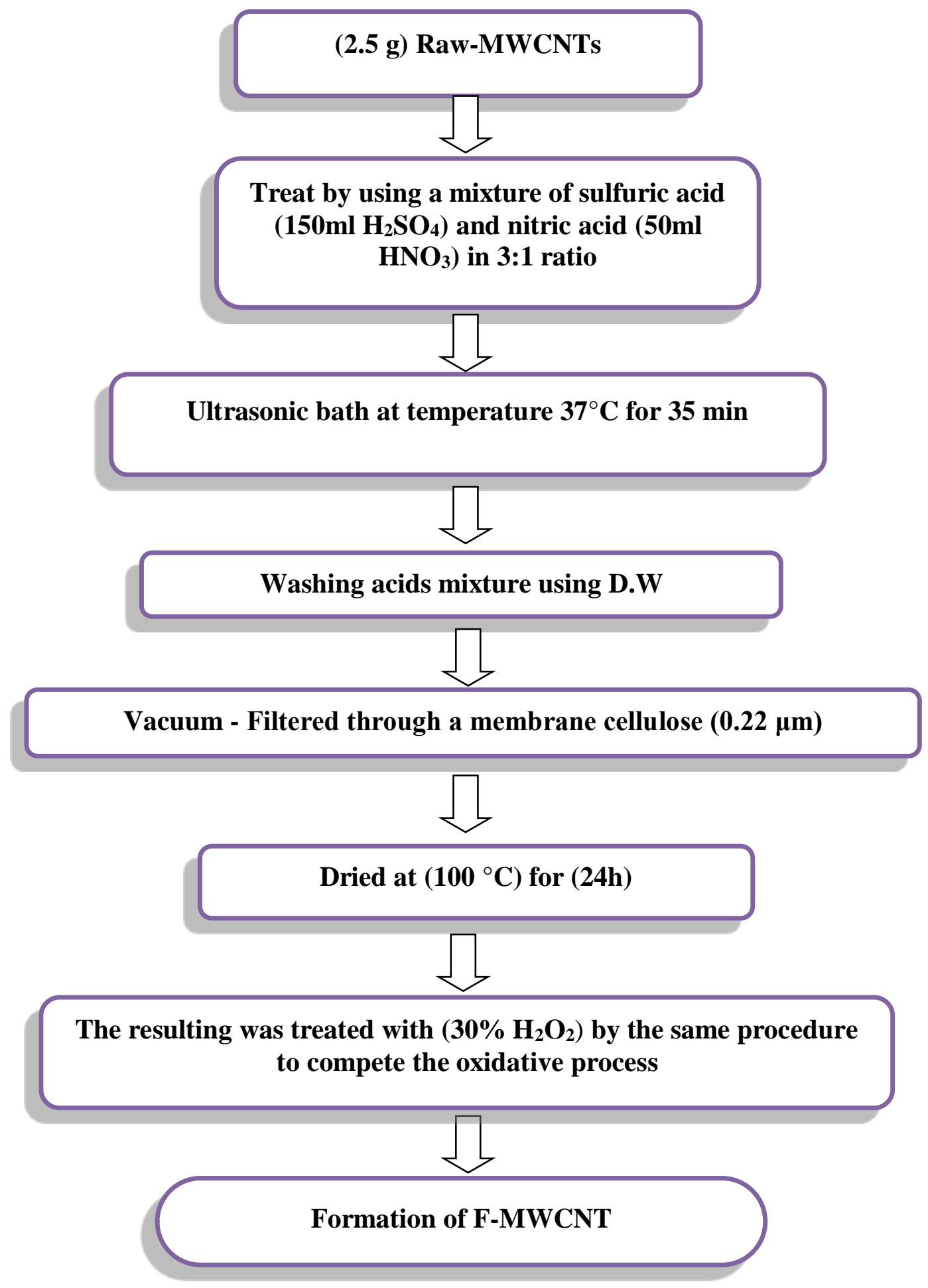

Figure 1: Schematic diagram of functionalized F-MWCNTs by using an acid treatment.

\subsection{Material characterization}

Multi- walled carbon nanotubes (purity $>95 \mathrm{wt} \%$; diameter $\sim 8-15 \mathrm{~nm}$; length $\sim 10-50 \mu \mathrm{m}$; Ash $<1.5 \mathrm{wt} \%$, Cheaptubes Grafton, USA) were all commercially purchased Sulfuric acid, J.T. Baker, USA. Nitric acid (CDH, India), Hydrogen peroxide (GCC, China) and Cellulose nitrate filter $(0.22 \mu \mathrm{m}$, Sartorius, all solutions were used directly without primary decontamination. The crystal structure and grain size before and after treating the MWCNTs were identified by X-ray technology (XRD-6000, Shimadzu) with $\mathrm{Cu} \mathrm{K \alpha}$ radiation source (wavelength 


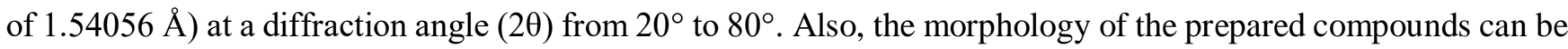
identified by field emission scanning electron microscopy (FE-SEM, Mira 3-XMU) and quickening voltage (20$30 \mathrm{kV}$ ). The surface charge of the nanostructures was studied using Zeta potential (Zeta Malvern ZS 100, Zetasizer).

\subsection{Inhibition Zone test (IZ)}

The test of the effect by measuring the inhibition zone on agar plates was achieved by using a well diffusion method to determine the (IZ) of different prepared samples like MWCNTs and F-MWCNTs, respectively against Gramnegative (E. coli) and Gram-positive ( $S$. aureus) bacteria. Three different concentrations of each sample are used $(500,750,1000 \mu \mathrm{g} \backslash \mathrm{ml})$ against the bacteria. The activated bacteria culture (S. aureus and $E$. coli) was subcultured on nutrient agar at $37^{\circ} \mathrm{C}$ overnight, and then the suspension of bacteria was in normal saline (0.9\%wlv) to prepare initial concentration of $10^{7}-10^{8} \mathrm{CFU} / \mathrm{ml}$ of each bacteria strain using (standard McFarland tube 0.5). Each culture spreads on nutrient agar plates. Then, different concentration $(500,750$, and 1000$) \mu \mathrm{g} / \mathrm{ml}$ of each sample was poured into well in the whole plates and incubated over night at $37^{\circ} \mathrm{C}$. The inhibition zone diameter of each sample was measured in millimeter; also, the average of inhibition zones around three wells was determined.

\section{Results and Discussion}

Figure 2 represented the XRD patterns of R-MWCNTs and F-MWCNTs, respectively. The appearance of a broad peak of high-intensity at $2 \theta=25.8^{\circ}$ appears to be congruent to the interlayer distance of $3.43 \AA$. Also, there is a diffraction peak with low intensity at $2 \theta=44^{\circ}$ concerning the metal oxides contained in MWCNTs that have been applied as a catalyst in the MWCNTs formation method. Both peaks are attributed to the hkl planes of (002), (100), respectively (JCPDS No. 01-0646). After treatment of acid the XRD patterns of F-MWCNTs, show a broad diffraction peak at $2 \theta=25.7^{\circ}$ corresponding to the interlayer distance of $3.45 \mathrm{~A}^{\circ}$ and a low intensity diffraction peak at $2 \theta=43.20^{\circ}$ corresponding to the interlayer distance of $2.07 \AA$. Both peaks are a duo to the hkl planes of (002), (100), respectively. A significant shift was observed in the diffraction peak F-MWCNTs due to the appearance of functional oxygen groups that increase the interlayer distance between the F-MWCNTs layers. This specify the success of the functional process using the acid treatment without changing the structure of the FMWCNTs. and, the peaks of catalysts disappeared after acid treatment of MWCNTs $[4,17,18]$.

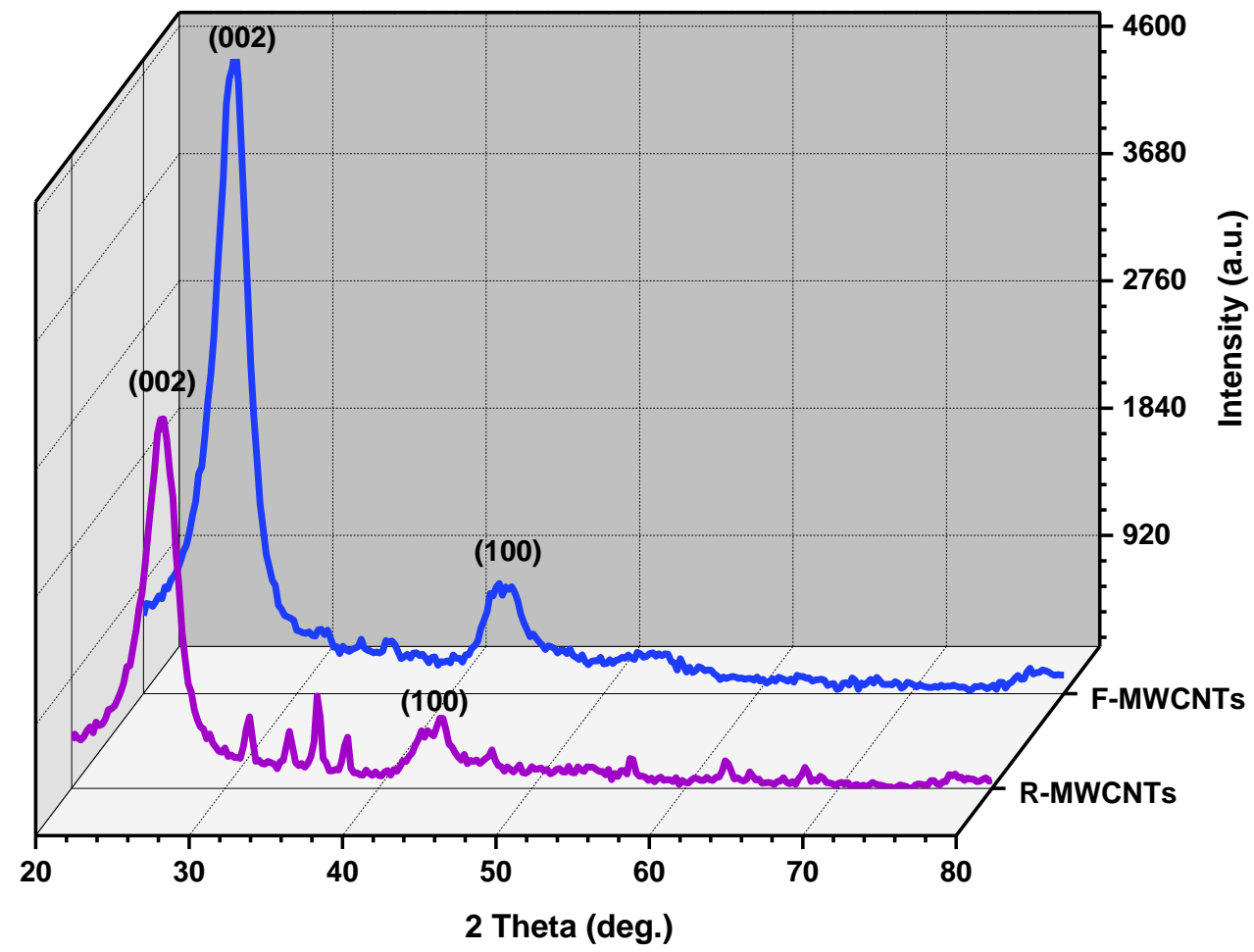

Figure 2: XRD patterns for R-MWCNTs and F-MWCNTs samples. 
Moreover, the average crystalline size is calculated by using the Scherer's equation. It observes that the average crystal size of the R-MWCNTs and F-MWCNTs are about (3.27 and 3.19) nm, respectively. the average crystal size of high intensity peaks calculated from the Scherer's equation (1) is about $25.43 \mathrm{~nm}$ [19-21].

$$
D=K \lambda \backslash \beta \cos \theta
$$

Where (D) is crystallite sizes, $(\mathrm{k})$ is a constant and equal to 0.9 and $(\beta)$ is a full width at half maximum (FWHM) of the diffraction peak.

The FE-SEM images of R-MWCNTs before the acid treatment with different modifications were confirmed as shown in Figure 3 a, 3 b. It was observed that the R- MWCNTs appear as cylindrical shaped tubes in different directions. Also, the tubes tend to aggregate more due to Van der Waals forces between the tubes. The state of the acid treatment of F-MWCNTs, it is observed that there is a significant difference in the FE-SEM analysis as shown in Figure 3 a, $3 \mathrm{~b}$. The microscopic images explain that the nanotubes appear in the form of complex tubes that look like ropes. The F-MWCNTs tubes appear shorter in length and have less aggregation compared to the RMWCNTs. Also, F-MWCNTs structure shows an anomaly with defects on the surface of tubes. The average tube diameters of R-MWCNTs and F-MWCNTs are about 29.59nm and 34.62nm, respectively[22, 23]. The results obtained from the FE-SEM analysis are similar to a study prepared by Karthikeya et al [24].
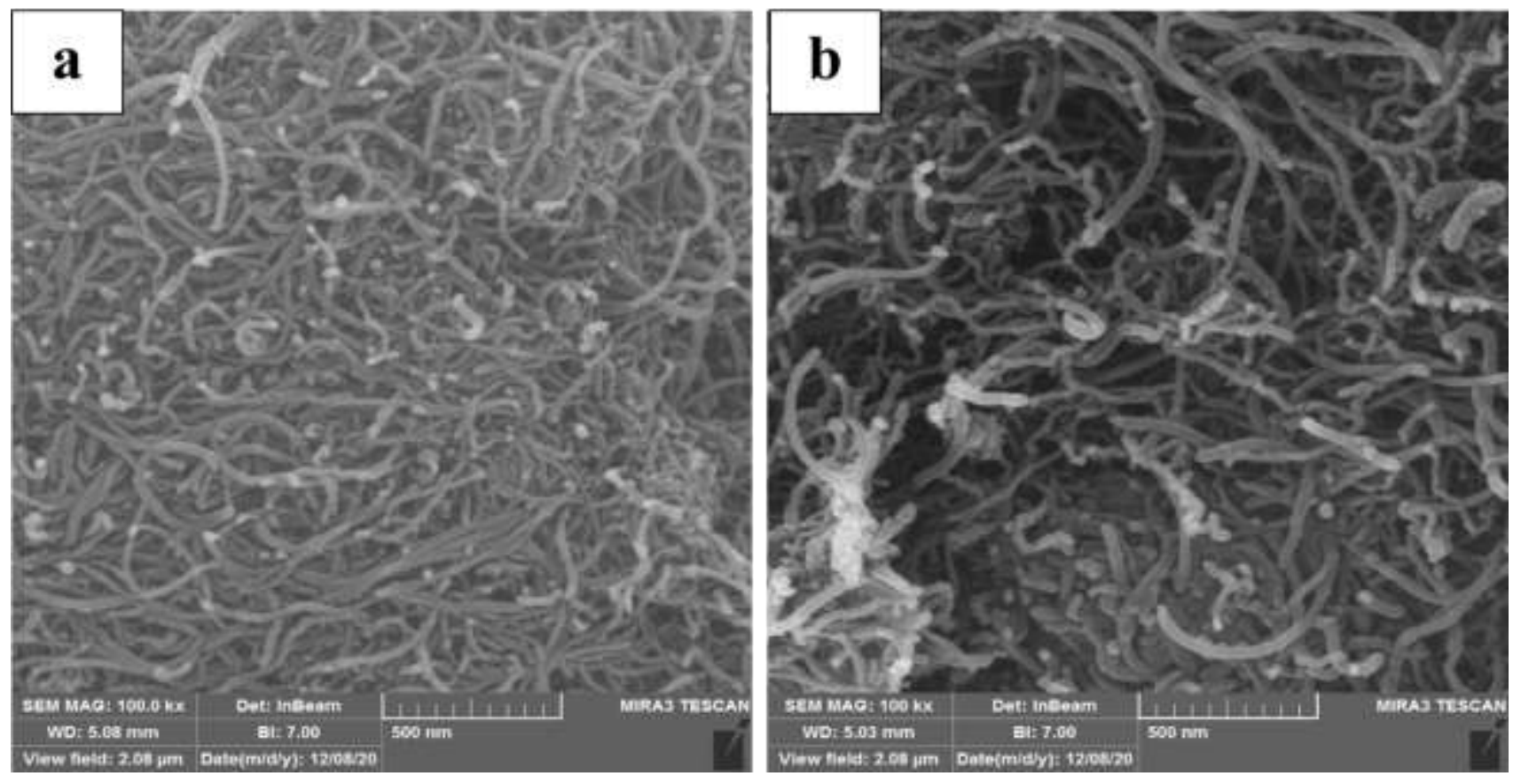

Figure 3: FESEM images of a) R-MWCNTs and b) F-MWCNTs.

The qualitative analysis of stability and symmetry of R-MWCNTs and F-MWCNTs samples were determined by examining the zeta potential as shown in Figure 4. The zeta potential measurement of the R-MWCNTs shows a low negative value is of $-9 \mathrm{mV}$. This indicates of a decrease in the colloid stability for the solution due to the cubulation of suspended nanoparticles. 


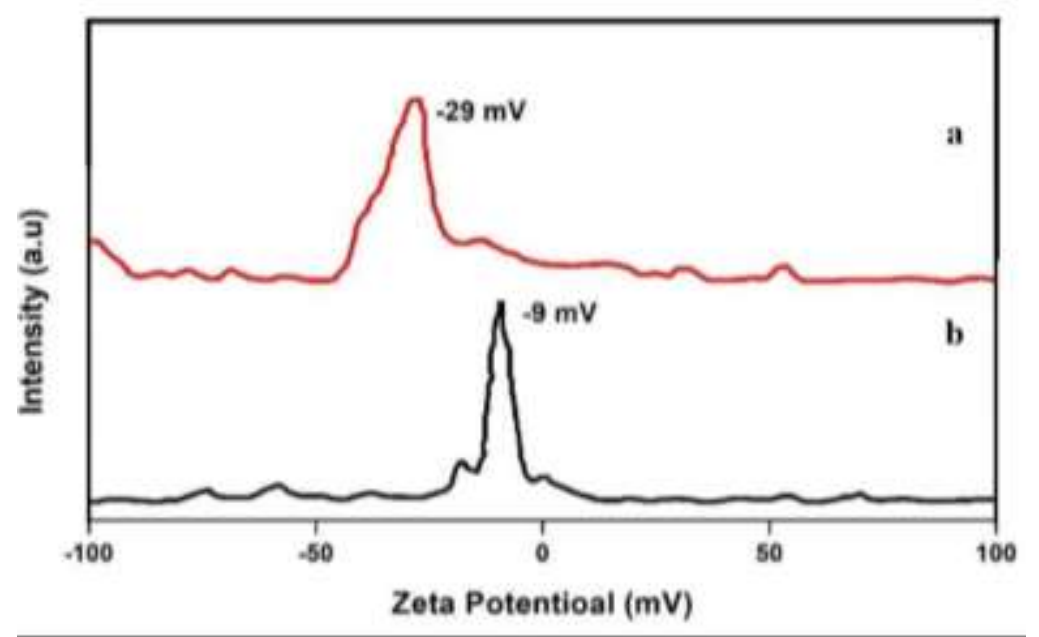

Figure 4: Zeta potential distributions curves for a) F-MWCNTS b)R-MWCNTS.

After the functionalization method of F-MWCNTs, the zeta potential value increased up to $-29 \mathrm{mV}$. This respectable difference in the zeta potential is related to the definition of functional oxygen groups (negatively charged like $-\mathrm{OH}$ and $-\mathrm{COOH}$ ) on the outer and inner walls of the F-MWCNT due to the acid treatment. These in turn, increase the electrostatic aversion between the nanotubes, which makes the F-MWCNT able to disband in polar solutions. In contrast, these results reveal that the F-MWCNTs have good physical stability due to the high electrostatic aversion in polar solvents such as ethanol. The study is similar to the previous literature reported by Haider J et al [25]. Sayanti B et al[26], and Ahmed D et al [27]. The antibacterial activity of R-MWCNTs and FMWCNTs was tested by using the well diffusion method against gram-negative (E. coli ) and gram-positive (S.aureus ) bacteria as shown in Figure 5 and Table 1 as well as Figure 6, and Table 2, which largely use for quick antibiotic capability determination[28-30].

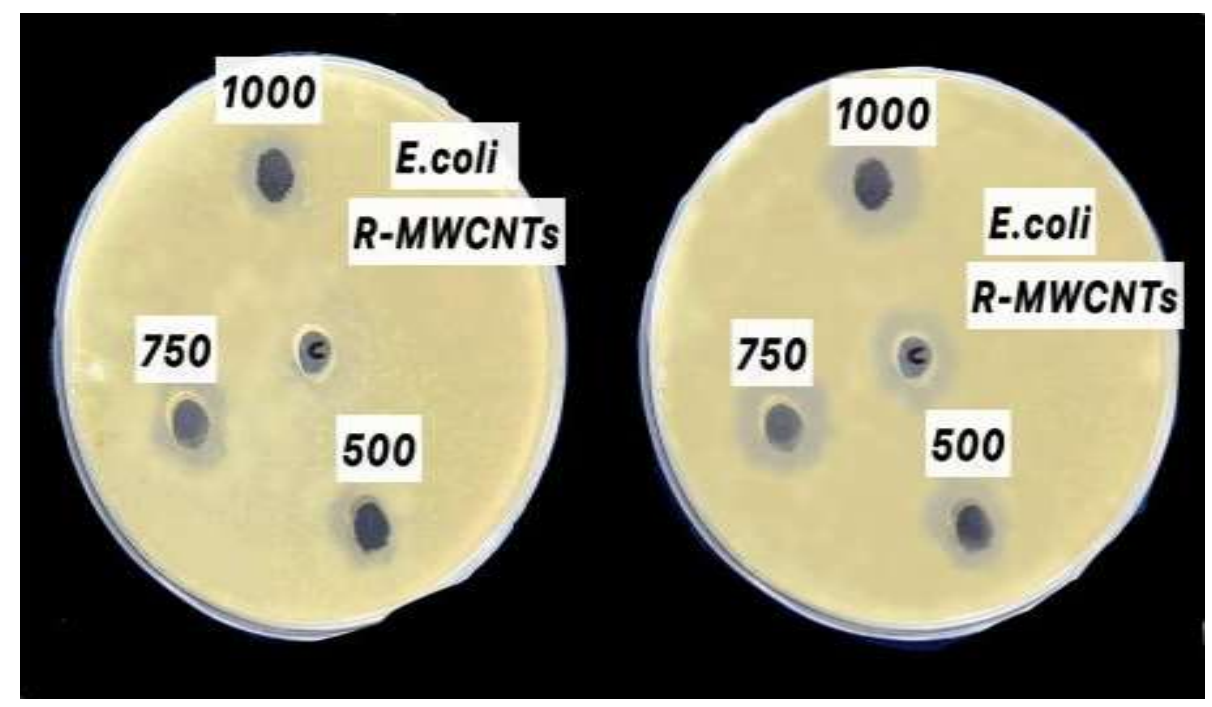

Figure 5: Images of Inhibition Zone diameter (IZ) with different concentrations of antibacterial R-MWCNTs against $S$. aureus and E. coli and b) the table of (IZ) against $S$. aureus and E. coli. 
Table 1: Inhibition Zone diameter (IZ) with different concentrations of antibacterial R-MWCNTs against S. aureus and E. coli and b) the table of (IZ) against S. aureus and E. coli.

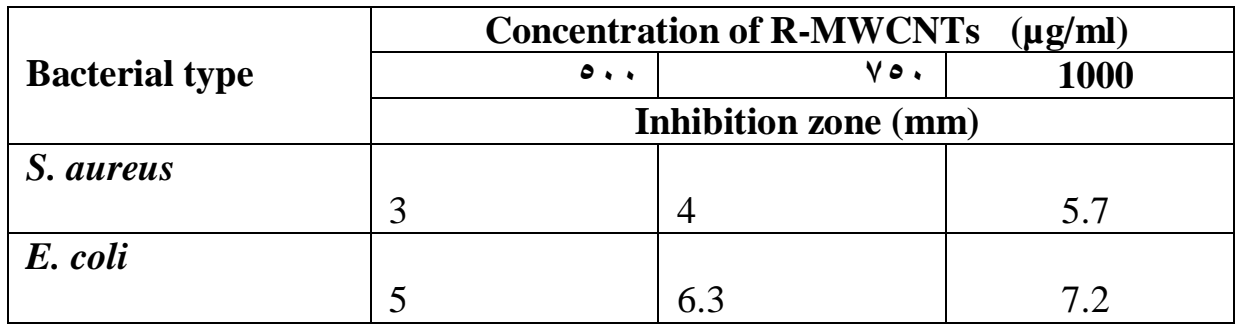

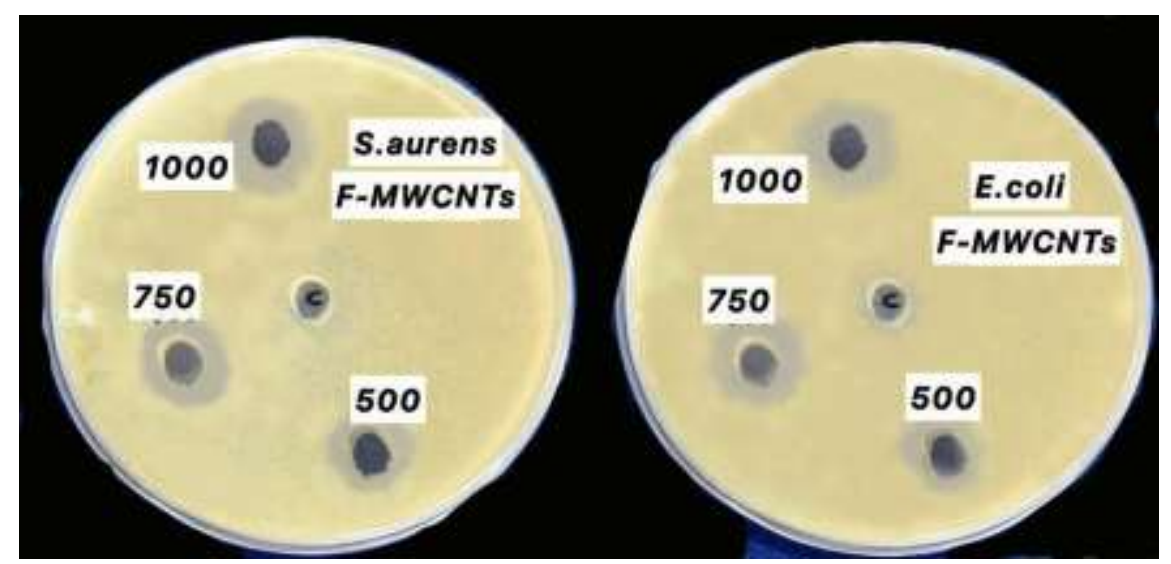

Figure 6: Images of Inhibition Zone diameter (IZ) with different concentrations of antibacterial F-MWCNTs against $S$. aureus and E. coli.

Table 2: Inhibition Zone diameter (IZ) with different concentrations of antibacterial F-MWCNTs against $S$. aureus and E. coli.

\begin{tabular}{|l|c|l|c|}
\hline \multirow{2}{*}{ Bacterial type } & \multicolumn{3}{|c|}{ Concentration of F-MWCNTs $(\boldsymbol{\mu g} / \mathbf{m l})$} \\
\cline { 2 - 4 } & 0. & Vo. & 1000 \\
\cline { 2 - 4 } & \multicolumn{3}{|c|}{ Inhibition zone (mm) } \\
\hline S. aureus & 7.2 & 7.8 & 12.3 \\
\hline coli & 10.0 & 12.8 & 16.3 \\
\hline
\end{tabular}

\section{Conclusions}

Using the acid treatment, MWCNTs and F-MWCNTs were modified. Then, the effect of the change was studied. $\mathrm{X}$-ray diffraction showed that the shift was observed in the diffraction peak F-MWCNTs due to the presence of functional oxygen groups that increase the interlayer distance between the F-MWCNTs layers. This indicates the success of the functional process using sulfuric acid and nitric acid without changing the structure of the FMWCNTs. Besides, the peaks of catalysts disappeared after acid treatment of MWCNTs. The FE-SEM images of the F-MWCNTs tubes appear shorter in length and have less agglomeration compared to the R- MWCNTs; Also, F-MWCNTs structure shows irregularity with defects on the surface of the tubes, the zeta observed that the prepared samples had good stability. These results reveal that the F-MWCNTs have good physical stability due to the high electrostatic repulsion in polar solvents such as a high zeta potential value up to $(-9,-29) \mathrm{mV}$ for MWCNTs and F-MWCNTs respectively. The study of the antibacterial activity of the R-MWCNTs and F-MWCNTs showed a strong activity that gradually increased with the increasing concentration toward $S$. aureus and E. coli. 


\section{Acknowledgments}

We are grateful to Medical Lab. Technique in Al-Esraa University Collage for their support of the work by making the biological tests in the Labs and providing us the protocol of making these tests.

\section{Conflict of Interest}

The authors declare that they have no conflict of interest.

\section{References}

[1] S. Anwar, M. Y. A. Raja, S. Qazi et al., Nanotechnology for telecommunications: Crc Press, 2017.

[2] M. Kröger, S. Hamwi, J. Meyer et al., "P-type doping of organic wide band gap materials by transition metal oxides: A case-study on Molybdenum trioxide," Organic Electronics, vol. 10, pp. 932-938, 2009.

[3] N. K. Mehra, A. K. Jain, N. Lodhi et al., "Challenges in the use of carbon nanotubes for biomedical applications," Critical Reviews ${ }^{\text {TM }}$ in Therapeutic Drug Carrier Systems, vol. 25, pp. 169-206, 2008-05-02 2008.

[4] D. Ahmed, and A. Abed, "Controlled surface modification of CNTs using mild acids through powerful sonication technique," Acta Physica Polonica, vol. 134, pp. 7-9, 2018.

[5] H. He, L. A. Pham-Huy, P. Dramou et al., "Carbon nanotubes: applications in pharmacy and medicine," BioMed research international, vol. 2013, p. 578290, 2013.

[6] D. S. Ahmed, A. J. Haider, and M. Mohammad, "Comparesion of functionalization of multi-walled carbon nanotubes treated by oil olive and nitric acid and their characterization," Energy Procedia, vol. 36, pp. 1111-1118, 2013.

[7] Z. Liu, S. M. Tabakman, Z. Chen et al., "Preparation of carbon nanotube bioconjugates for biomedical applications," Nature protocols, vol. 4, pp. 1372-1381, 2009.

[8] R. Mohammad, "Duha S. Ahmed, Mustafa KA Mohammed \& Mohammad," Chem. Pap, vol. 74, pp. $197-$ 208, 2020.

[9] V. Rastogi, P. Yadav, S. S. Bhattacharya et al., "Carbon nanotubes: an emerging drug carrier for targeting cancer cells," Journal of drug delivery, vol. 2014, pp. 670815,2014.

[10] M.-I. Baraton, "Nano- $\mathrm{TiO}_{2}$ for solar cells and photocatalytic water splitting: scientific and technological challenges for commercialization," The Open Nanoscience Journal, vol. 5, pp. 64-77,2011.

[11] Z. Xia, P. Guduru, and W. Curtin, "Enhancing mechanical properties of multiwall carbon nanotubes via s p 3 interwall bridging," Physical review letters, vol. 98, pp. 245501, 2007.

[12] E. Uc-Cayetano, F. Avilés, J. Cauich-Rodríguez et al., "Influence of nanotube physicochemical properties on the decoration of multiwall carbon nanotubes with magnetic particles," Journal of nanoparticle research, vol. 16, pp. 2192, 2014.

[13] E. Uc-Cayetano, F. Avilés, J. Cauich-Rodríguez et al., "Influence of nanotube physicochemical properties on the decoration of multiwall carbon nanotubes with magnetic particles," Journal of nanoparticle research, vol. 16, pp. 1-13, 2014.

[14] S. R. Paital, and N. B. Dahotre, "Calcium phosphate coatings for bio-implant applications: Materials, performance factors, and methodologies," Materials Science and Engineering: R: Reports, vol. 66, pp. 170, 2009.

[15] G. Manivasagam, D. Dhinasekaran, and A. Rajamanickam, "Biomedical implants: corrosion and its prevention-a review," Recent patents on corrosion science, vol. 5, pp. 40-45, 2010.

[16] S. M. Al-Jawad, M. R. Mohammad, and N. J. Imran, "Effect of electrolyte solution on structural and optical properties of $\mathrm{TiO}_{2}$ grown by anodization technique for photoelectrocatalytic application," Surface Review and Letters, vol. 25, pp. 1850078, 2018.

[17] A. J. Haider, M. R. Mohammed, and D. S. Ahmed, "Influence of functionalization MWCNTs using acid treatment on Gram negative and Gram positive bacteria," Iraqi Journal of Applied Physics, vol. 10, pp. 29-33, 2014. 
[18] S. M. AL-Jawad, A. A. Taha, and A. M. Redha, "Studying the structural, morphological, and optical properties of $\mathrm{CuS}$ : Ni nanostructure prepared by a hydrothermal method for biological activity," Journal of Sol-Gel Science and Technology, vol. 91, pp. 310-323, 2019.

[19] G. Allaedini, P. Aminayi, and S. M. Tasirin, "Structural properties and optical characterization of flowerlike $\mathrm{Mg}$ doped NiO,” AIP Advances, vol. 5, pp. 077161, 2015.

[20] S. M. Al-Jawad, "Influence of multilayer deposition on characteristics of nanocrystalline $\mathrm{SnO}_{2}$ thin films produce by sol-gel technique for gas sensor application," Optik, vol. 146, pp. 17-26, 2017.

[21] S. M. Al-Jawad, S. H. Sabeeh, A. A. Taha et al., "Synthesis and characterization of Fe-ZnO thin films for antimicrobial activity," Surface review and letters, vol. 26, pp. 1850197, 2019.

[22] A. Zaman, T. U. Rashid, M. A. Khan et al., "Preparation and characterization of multiwall carbon nanotube (MWCNT) reinforced chitosan nanocomposites: effect of gamma radiation," BioNanoScience, vol. 5, pp. 31-38, 2015.

[23] G. R. Yerawar, "Characterization of Chemically Synthesized Polyaniline-Zinc Oxide Nanocomposites," Der Pharma Chemica, vol. 4, pp. 1288-1291, 2012.

[24] P. V. Kumar, A. J. Ahamed, and A. Ravikumar, "Synthesis of $\mathrm{Mg}^{2+}$ doped $\mathrm{NiO}$ nanoparticles and their structural and optical properties by Co-precipitation method," Journal Of Advanced Applied Scientific Research, vol. 2, pp. 1-9, 2020.

[25] A. J. Haider, A. D. Thamir, D. S. Ahmed, and M. R. Mohammad, "Deposition of silver nanoparticles on multiwalled carbon nanotubes by chemical reduction process and their antimicrobial effects," AIP Conference Proceedings, vol. 1758, pp. 030003, 2016.

[26] S. Brahmachari, S. K. Mandal, and P. K. Das, "Fabrication of SWCNT-Ag nanoparticle hybrid included self-assemblies for antibacterial applications," PLOS One, vol. 9, pp. e106775, 2014.

[27] D. S. Ahmed, and M. K. Mohammed, "Studying the bactericidal ability and biocompatibility of gold and gold oxide nanoparticles decorating on multi-wall carbon nanotubes," Chemical Papers, vol. 74, pp. 40334046, 2020.

[28] A. A. Taha, A. D. Faisal, S. Yousif et al., "Antimicrobial Activity of Locally Synthesized Carbon Nanosphere on Some Pathogenic Species of Bacteria and Parasites," Baghdad Science Journal, vol. 18, pp. 1-6, 2021.

[29] M. A Al-Kinani, A. Haider Sharafaldin Al-Musawi., "Study the Effect of Laser Wavelength on Polymeric Metallic Nanocarrier Synthesis for Curcumin Delivery in Prostate Cancer Therapy: In Vitro Study," Journal of Applied Sciences and Nanotechnology, vol. 1, pp.43-5, 2021.

[30] N. Hussein M., Khadum., "Evaluation of the Biosynthesized Silver Nanoparticles" Effects on Biofilm Formation," Journal of Applied Sciences and Nanotechnology, vol. 1, pp.23-31,2021. 